\title{
X-ray and optical orbital modulation of EXO 0748-676 : A co-variability study using XMM-Newton ${ }^{*}$
}

\author{
Gayathri Raman ${ }^{\mathrm{a}, * *}$, Biswajit Paul $^{\mathrm{a}}$ \\ ${ }^{a}$ Raman Research Institute, C. V. Raman Avenue, Sadashivanagar, Bangalore-560012, India
}

\begin{abstract}
'Abstract
We present a multi-wavelength timing study of the eclipsing low mass X-ray binary EXO 0748-676 (UY Vol) using XMM-Newton when the source was in a hard spectral state. The orbital optical and X-ray light curves show a fairly large amount of intensity modulation in the 7 observations taken during September-November, 2003, covering 36 complete binary orbits of EXO 0748-676. While assessing the non-burst variability, simultaneously in the optical and X-ray light curves, we find that they are not correlated at reprocessing or orbital time-scales, but are weakly correlated at a few 1000s of seconds time-scales. Although a large fraction of the optical emission is likely to be due to reprocessing, the lack of significant correlation and presence of large variability in the orbital X-ray and optical light curves is probably due to structures and structural changes in the accretion disk that produce, and sometimes mask the reprocessed signal in varying amounts. These disk structures could be induced, at least partly, by irradiation. From the observed modulations seen in the optical light curves, there is strong evidence of accretion disk evolution at time scales of a few hours.
\end{abstract}

Keywords: binaries: eclipsing, stars: individual: EXO 0748-676, stars: neutron, X-rays

\section{Introduction}

EXO 0748-676 is a low mass X-ray binary (LMXB) that was discovered with EXOSAT in 1985 (Parmar et al., 1986). Soon after its discovery the optical counterpart was identified as UY Vol (Wade et al., 1985; ; Pedersen et al., 1985). It is a high inclination $\left(\sim 75-82^{\circ}\right)$ eclipsing binary system with an orbital period of $3.82 \mathrm{hr}$ (Parmar et al., 1985; Crampton et al., 1986; Parmar et al., 1986). The presence of thermonuclear bursts in the X-ray light curve established the compact object in the binary as a neutron star (Gottwald et al., 1986; Parmar et al., 1986). Many high and low frequency variabilities have been detected in this source, including a Quasi Periodic Oscillation (QPO) at $695 \mathrm{~Hz}$ (Homan and van der Klis, 2000). Galloway et al. (2010) discovered burst oscillations at $552 \mathrm{~Hz}$, which is likely to be the spin period of the neutron star in EXO 0748-676. This object also shows pre-eclipse intensity dips that are commonly understood to be due to obscuration of the central X-ray source by structures in the outer disk, whose azimuthal distribution is variable (Parmar et al., 1986). Mass of the companion has been estimated to be in the range of 0.16 to $0.42 \mathrm{M}_{\odot}$ with the upper limit corresponding to an M2V spectral type star (Hynes and Jones, 2009).

In addition to eclipses, dips and thermonuclear $\mathrm{X}$-ray bursts, this system exhibits a lot of intensity modulation in the X-rays

\footnotetext{
*based on observations obtained with XMM-Newton, an ESA science mission with instruments and contributions directly funded by ESA Member States and NASA.

**Corresponding author: Gayathri Raman; Email:graman@rri.res.in
}

as well as in the UV/optical (Crampton et al., 1986; Parmar et al., 1986; van Paradijs et al., 1988). A significant fraction of the UV/optical emission is understood to be reprocessed emission from the accretion disk (van Paradijs et al., 1988). The broad and shallow eclipses seen in the optical as against the narrow and sharp X-ray eclipses indicate that the reprocessed optical photons are being emitted from an extended region, namely, the accretion disk (Crampton et al., 1986; van Paradijs et al., 1988).

EXO 0748-676 was moderately bright and displayed a lot of short term variability since the time of its discovery in 1985 till 2008. For a span 24 years, its persistent X-ray luminosity remained at $\sim 10^{36-37} \mathrm{ergs} / \mathrm{s}$ (Degenaar et al., 2011), after which, it went into quiescence in 2008 (Hynes and Jones, 2009).

Recently, a number of studies involving high inclination neutron star X-ray binaries indicate a correlation between the luminosity state and disk-wind outflows (Ponti et al., 2014). This is similar to the correlation between spectral state and radio jet outflows observed in black hole binaries (Ponti et al., 2012). EXO 0748-676 and AX J1745-281 are two systems in which highly ionized Fe absorption lines (Fe XXV and Fe XXVI) were detected specifically during their soft spectral state (Ponti et al., 2014, 2015). These absorption lines, being indicators of diskwinds, confirmed the fact that a correlation between luminosity state and presence of disk-wind indeed exists (Ponti et al.,2014, 2015). There have also been instances of simultaneous detection of radio-jets as well as disk-wind outflow signatures from objects like GX 13+1, Sco X-1 and GX 340+0, in the hard luminosity state, which indicate that jets and disk-winds may not be mutually exclusive (Homan et al., 2016). 
Soon after its discovery, it was observed that EXO 0748-676 displayed different X-ray intensity states (Parmar et al., 1986; Gottwald et al., 1986). Recent studies by Ponti et al. (2014), showed that the bright state displayed less intense dipping phenomenon compared to the faint state. We have also explored this intensity state dependent dipping behavior of EXO 0748676 , in this current work.

Simultaneous X-ray and optical data have been used previously to study the co-variability in LMXBs. Some sources showed X-ray and optical correlation during quiescence (4U 1735-44: Corbet et al. 1989, V404 Cyg: Hynes et al. 2004); some, no correlation during quiescence (Cen X-3: Cackett et al. 2013); others exhibit correlation during highest emission (4U 1556-605: Motch et al. 1989a); while anti-correlated emission (4U 0614+09: Machin et al. 1990) or even bimodal behavior (i.e., correlation as well as anti-correlation, Sco X-1: Ilovaisky et al 1980) is also seen. The lack of a consistent relation between $\mathrm{X}$-ray and optical variations in LMXBs have usually been interpreted to be due to changes in the extent and visibility of the reprocessing structures that produce most of the optical variability.

The X-ray and optical emission for EXO 0748-676 was initially seen on an average to be correlated on short time scales (Motch et al., 1989b). Thomas et al. (1993) studied the correlated X-ray and optical flux in EXO 0748-676 to understand the reprocessed emission. They did not find any X-ray and optical co-variability that was indicated from earlier studies. Southwell et al. (1996) also confirmed this change in the nature of co-variability. In fact, a larger X-ray flux during the bright state would mean an increased mass accretion rate that should make the outer disk bulge more prominent, thereby giving rise to the broad optical eclipse or the optical high state. The lack of X-ray and optical co-variability was associated with the geometric masking of the reprocessed emission. Understanding how the system geometry alters the X-ray and reprocessed optical signal requires correlation studies, which we have carried out with very long, simultaneous X-ray and optical light curves, obtained using XMM-Newton.

In this paper, we probe the non-burst co-variability of the $\mathrm{X}$ rays and optical emission in this quasi-persistent binary, using XMM-Newton observations, when it was in the hard state in 2003. We also present the orbit-to-orbit variations seen in the $\mathrm{X}$-ray and optical light curves.

\section{Observations}

EXO 0748-676 was observed during September-November, 2003 with XMM-Newton. Details of the archival data involving 7 Obs-IDs are shown in Table 1. We have used data from the European Photon Imaging Camera (EPIC) instrument using the PN CCD $(0.1-10 \mathrm{keV})$ for all of the 7 observations. The Optical Monitor (OM, Mason et al. 2001) was simultaneously operated in timing mode using the white band $(170-650 \mathrm{~nm})$.
EXO 0748-676 was observed for $\sim 600 \mathrm{ks}$ spanning all the 7 Obs-IDs in the X-rays. The EPIC-PN and OM together thus provide a simultaneous multi-wavelength coverage to the target in the X-ray and UV/optical bands.

We reduced the EPIC-PN data using the SAS version 12.0.1 software, using the latest calibration files. The X-ray light curves were extracted in the soft $(0.3-5 \mathrm{keV})$ and hard $(5-10 \mathrm{keV})$ bands from a 40" radius centered on the position of EXO 0748676 with $1 \mathrm{~s}$ binning. The optical light curve was extracted from a region of 6" around the source using the omichain task which processes the data with the latest calibration files, and subsequently performs source detection and aperture photometry.

There have been previous burst studies using this dataset by Boirin et al. (2007), spectroscopic studies of the dips by Díaz Trigo et al. (2006), burst spectral studies by Cottam et al. (2008) and a com. parison of the optical to X-ray burst emission by Paul et al. (2012). During another observation of EXO 0748-676 with XMM-Newton in 2005 (Obs-ID 0212480501), the source was found to be in a soft spectral state (Ponti et al., 2014). We reduced the OM data for this particular dataset as well. However, because of the different filters used and relatively short exposures, the optical light curves from this observation were not suitable for further analysis.

\section{Data analysis and results}

\subsection{X-ray and optical light curves}

\subsubsection{Raw light curves}

The X-ray light curve has been divided into two bands, soft $(0.3-5.0 \mathrm{keV})$ and hard $(5.0-10.0 \mathrm{keV})$. A total of 76 thermonuclear X-ray bursts were reported from all the 7 observations in X-rays (Boirin et al., 2007), a summary of which is presented in Table 1. 63 of these bursts had simultaneous optical band data as well (Paul et al., 2012). There were gaps in the optical data during 13 of the X-ray bursts, because of which they were not recorded in the optical observations. Paul et al. (2012) fitted all the burst profiles with a model consisting of a constant component, a burst with a linear rise and an exponential decay. The X-ray and optical counts of the bursts were calculated by subtracting the pre-burst component from the total photon counts during the bursts. The bursts show an average $\mathrm{X}$-ray to optical conversion factor, which is the ratio of the optical counts to the X-ray counts for each of the burst, of 0.15 (Paul et al., 2012). Simultaneous X-ray and optical light curves for the Obs-ID 0160760201 are shown in Figure 1 with a bin size of $60 \mathrm{~s}$. Optical eclipses are broad depressions as opposed to the sharp and near total X-ray eclipses, indicating that a corresponding part of the optical emission originates in the inner accretion disk.

\subsubsection{Filtered non-burst light curves}

A time window file, created using the task xronwin, was used to exclude the thermonuclear burst time intervals from the $\mathrm{X}$-ray and optical light curves. The time windows, in some 


\begin{tabular}{|c|c|c|c|c|c|c|c|c|c|}
\hline Obs-ID & $\begin{array}{l}\text { Date } \\
\text { (dd/mm/yyyy) }\end{array}$ & $\begin{array}{l}\text { Start Time } \\
\text { UT (hh:mm) }\end{array}$ & $\mathrm{t}_{\text {exp }}(\mathrm{h})$ & $\begin{array}{l}\text { No. of } \\
\text { X-ray } \\
\text { bursts } \\
\end{array}$ & $\begin{array}{l}\text { No. of } \\
\text { Optical } \\
\text { bursts }\end{array}$ & $\begin{array}{l}\text { No. of } \\
\text { Full orbits }\end{array}$ & $\begin{array}{l}\text { Non-burst } \\
\text { soft X-ray } \\
\text { variability }(\%)\end{array}$ & $\begin{array}{l}\text { Non-burst } \\
\text { optical } \\
\text { variability }(\%)\end{array}$ & $\begin{array}{l}\text { Non-burst } \\
\text { hard X-ray } \\
\text { variability }(\%)\end{array}$ \\
\hline 0160760101 & $19 / 09 / 2003$ & $13: 37$ & 24.6 & 10 & 8 & 6 & 52.19 & 13.93 & 22.68 \\
\hline 0160760201 & $21 / 09 / 2003$ & $13: 38$ & 25.1 & 14 & 13 & 6 & 65.25 & 16.78 & 25.19 \\
\hline 0160760301 & $23 / 09 / 2003$ & $10: 42$ & 30.0 & 14 & 11 & 7 & 50.20 & 15.38 & 22.55 \\
\hline 0160760401 & $25 / 09 / 2003$ & $17: 29$ & 20.4 & 9 & 8 & 5 & 65.79 & 17.17 & 23.16 \\
\hline 0160760601 & $21 / 10 / 2003$ & $10: 02$ & 15.2 & 8 & 6 & 3 & 55.82 & 17.07 & 22.87 \\
\hline 0160760801 & $25 / 10 / 2003$ & $19: 19$ & 17.3 & 9 & 6 & 3 & 73.30 & 16.11 & 23.32 \\
\hline 01607601301 & $12 / 11 / 2003$ & $08: 24$ & 25.2 & 12 & 10 & 6 & 65.27 & 19.54 & 24.97 \\
\hline
\end{tabular}

Table 1: EXO 0748-676 was observed using XMM-Newton in 2003. This table details the Obs-IDs, date of observation, number of thermonuclear X-ray bursts, number or reprocessed optical bursts, number of complete orbital segments and non-burst r.m.s variability for the soft X-rays, hard X-rays and optical.

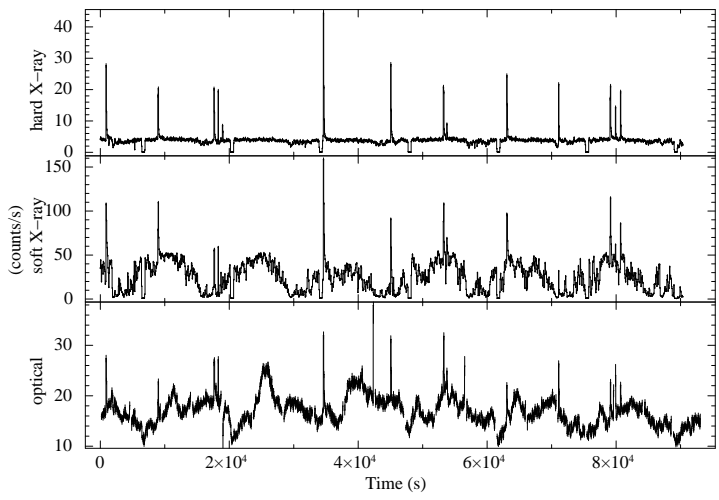

Figure 1: Simultaneous hard X-ray (5 - $10 \mathrm{keV})$, soft X-ray $(0.3-5.0 \mathrm{keV})$, and optical light curves with $60 \mathrm{~s}$ binning are shown for the Obs-ID 0160760201. The light curves show simultaneous thermonuclear bursts in all the three bands as well as significant non-burst intensity modulation in the soft X-ray and optical bands.

cases, contained a little more than just the X-ray burst duration to account for the delayed optical response and also the slow, extended decay tails. These time windows spanned a range of approximately 40 - $100 \mathrm{~s}$ depending on the duration of each of the bursts. Simultaneous hard X-ray, soft X-ray and optical light curves are shown in Figure 2 for all the 7 observations with a bin size of $60 \mathrm{~s}$. A significant amount of variability is seen in the soft X-ray band compared to the hard X-ray in all observations. The most prominent variability observed in the light curves is the orbital modulation. The pattern of orbital modulation changed significantly during the observations, which we discuss below. For almost all the Obs-IDs, the optical count rate varies between 10 - 25 counts/s and soft X-ray count rates are nearly $2-3$ times the optical rates. The percentage r.m.s variability with respect to the average values of the $60 \mathrm{~s}$ binned light curves in these bands for all the 7 observations are shown in Table 1. Significant differences in the intensity variation patterns like heavy to moderate dipping behavior, frequent short time-scale fluctuations, smooth orbital variability etc. are seen in all the three bands on different observation days.

\subsection{Orbit-to-orbit modulation}

To further study the sub-orbital short time-scale modulation and its evolution in consecutive orbits, the light curves were sliced into several orbits using the X-ray orbital ephemeris provided in Wolff et al. (2009). Plots containing soft X-ray and optical light curves from different binary orbits are overlaid and are shown in Figure 3 for all the seven observations.

The Obs-ID 0160760101 displays an erratic X-ray behavior with many soft X-ray dips at different phases. In four of the observations (Obs-IDs 0160760201, 0160760401, 0160760801 and 0160761301), pre-eclipse dips are seen in soft X-rays in the orbital phase range of 0.6-0.9. In some observations there are dips around phase 0.2 (Obs-ID 0160760601), 0.15 (ObsID 0160760401) and 0.45 (Obs-ID 0160761301). Other than the dips, most observations show large orbit-to-orbit intensity variations often by a factor of a few; for example at phase 0.2 in Obs-IDs 0160760201 and 0160760401 and at all phases in Obs-ID 0160760101. In Obs-ID 0160760601 there are dips in phase range 0.6-0.9 but with a large difference in the dips phases from orbit to orbit. The duty cycle of the soft X-ray dips is seen to be as high as $50 \%$ in some orbits.

Optical light curves show dips and even peaks at different phases. Obs-IDs 0160760201, 0160760301, 0160760401 and 0160760601 show peaking behavior at phases 0.1 and 0.4 with slight dips around phase 0.2 in some of the orbits. A single orbit each in Obs-IDs 0160760201 and 0160760601 peaks at phase 0.2 unlike the rest of the orbits. Obs-IDs 0160760101 and 0160760301 have a single peak around 0.25 , whereas ObsIDs 0160760801 and 0160761301 show a peak somewhere close to phase 0.3 . The soft $\mathrm{X}$-ray and corresponding optical variations are broadly classified into five classes according to the variable dipping seen in their orbital profiles (Figure 4). This classification is based on the observed soft X-ray light curves from all the 7 observations.

\subsection{Cross-correlation analysis}

We define three time-scales of variability to assess the correlation between soft, hard X-rays and optical emission. Reprocessing happens at extremely short time-scales, which are insignificant compared to the orbital time-scales. Probing such short time-scales is out of the scope of this work. The size of the accretion disk and the binary separation in EXO 0748-676 is about 3 light seconds (Hynes et al., 2006). However, other than the thermonuclear bursts, which have been removed from the current analysis, there is not much short time-scale (of the order of a few seconds) X-ray variability. Hence, we do not aim to investigate reprocessing at these time scales too. 
Obs ID 0160760101
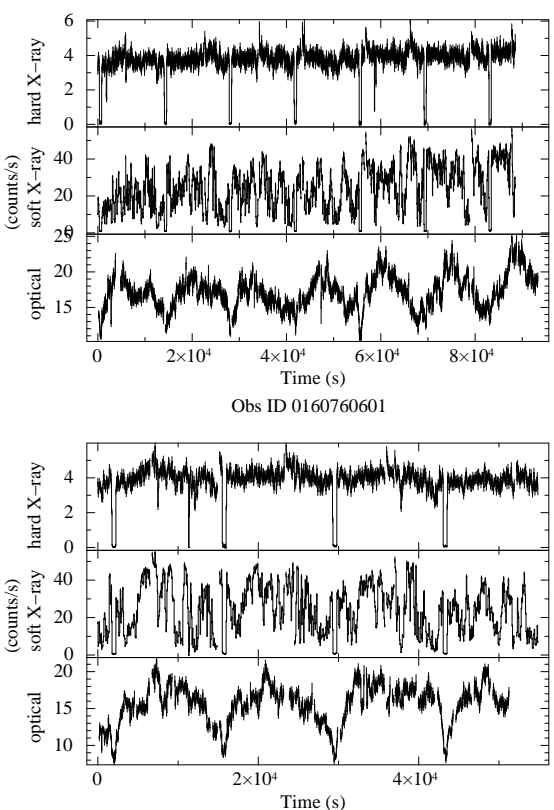

Obs ID 0160760401

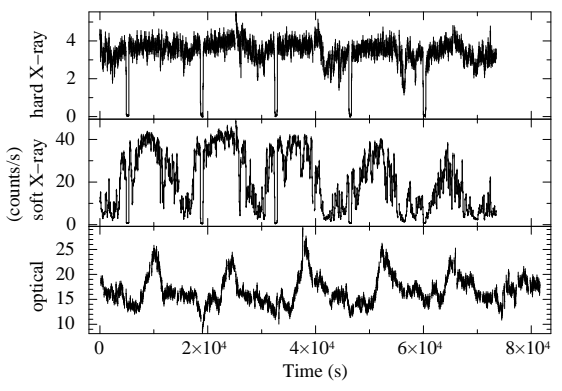

Obs ID 0160760301
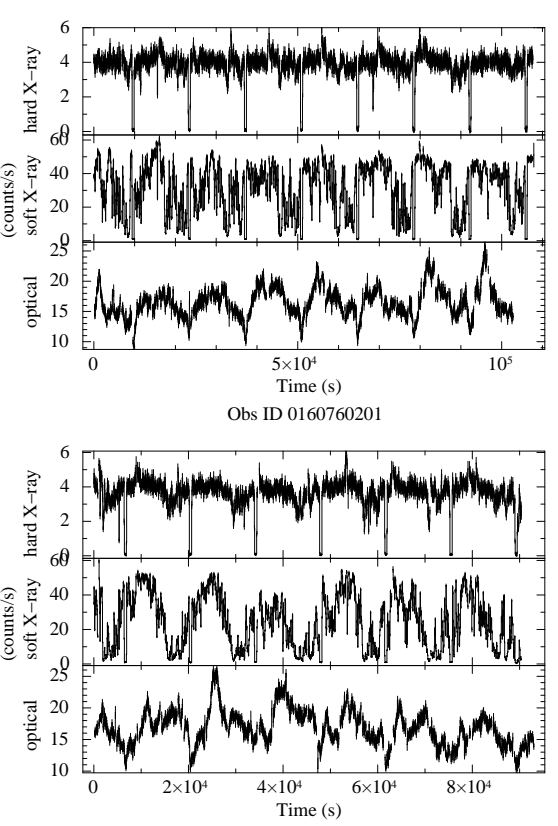

Obs ID 0160760801

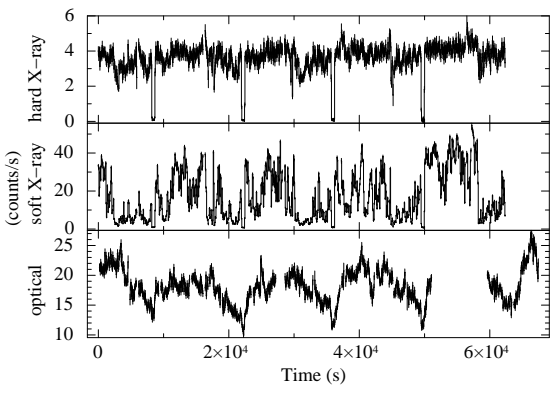

Obs ID 0160761301

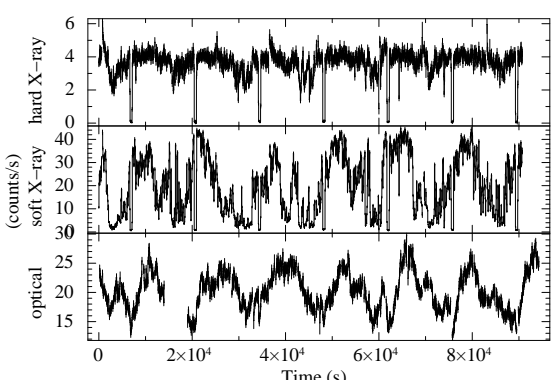

Figure 2: Simultaneous hard X-ray (5 - $10 \mathrm{keV})$, soft X-ray (0.3 - 5.0 keV), and optical light curves for all the Obs-IDs are shown with a filter applied to remove the thermonuclear burst sections. The non-burst X-ray and optical light curves display significant intensity modulation during all the observations. 
Obs-ID 0160760101

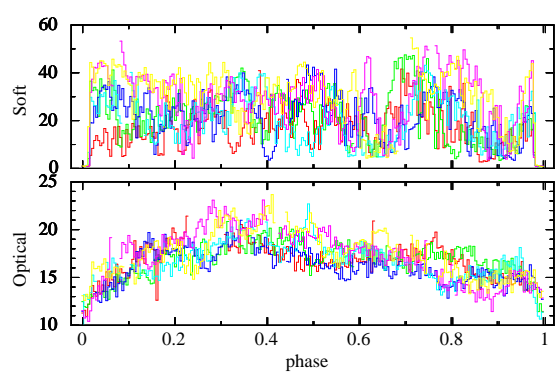

Obs-ID 016076030

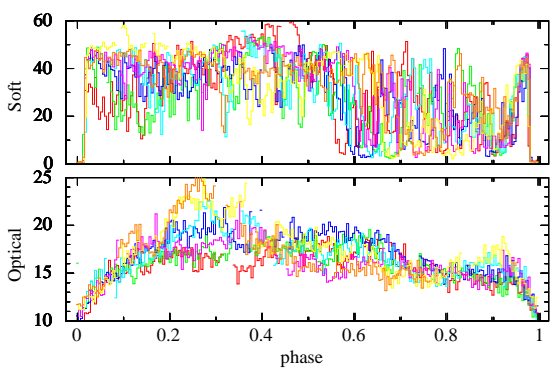

Obs-ID 0160760601

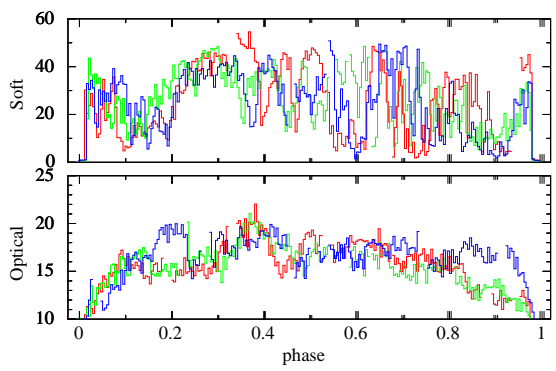

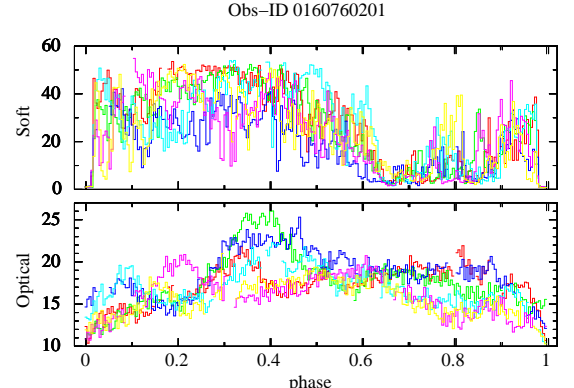

Obs-ID 0160760401

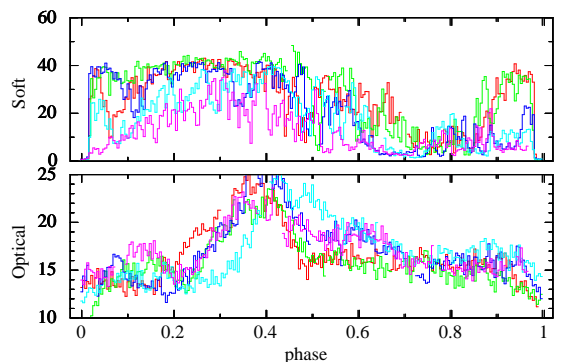

Obs-ID 0160760801

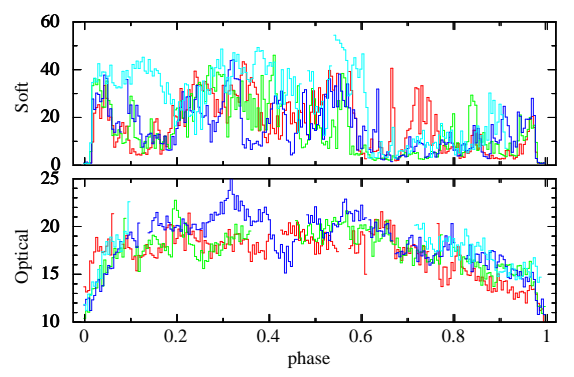

Obs-ID 0160761301

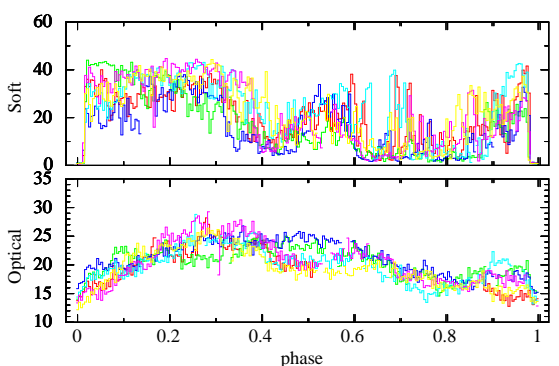

Figure 3: Simultanesou soft X-ray and optical orbit to orbit intensity modulations are shown for all the Obs-IDs . Each binary orbit is represented by a different color. The orbital light curve intensity is seen to vary enormously between successive binary orbits both in the soft X-rays and optical. 


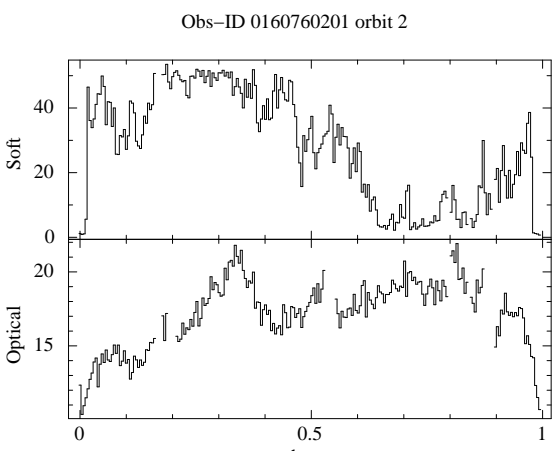

MJD Start time: 52903.64 Stop time: 52903.8
Obs-ID 0160760601 orbit 2

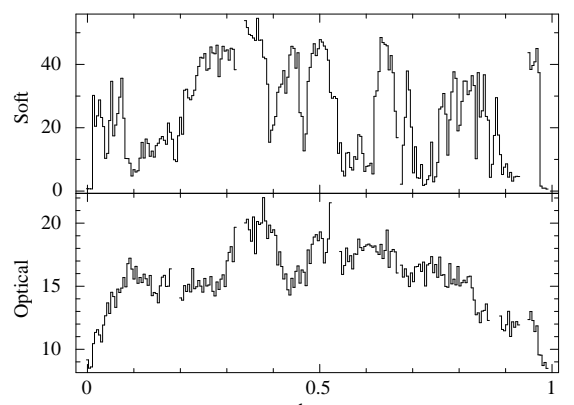

MJD Start time: 52933.44 Stop time: 52933.59 Obs-ID 0160761301 orbit 4

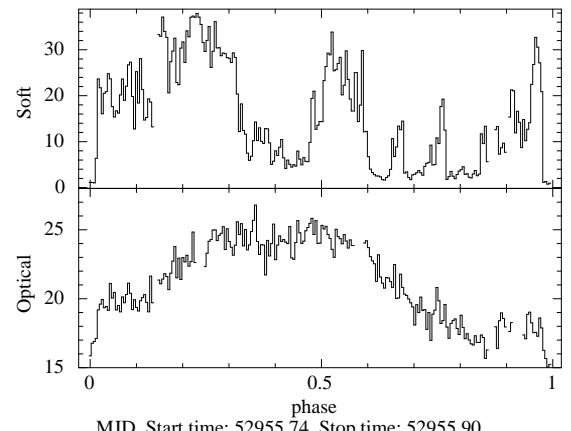

Obs-ID 0160760101 orbit 4

Obs-ID 0160761301 orbit 3
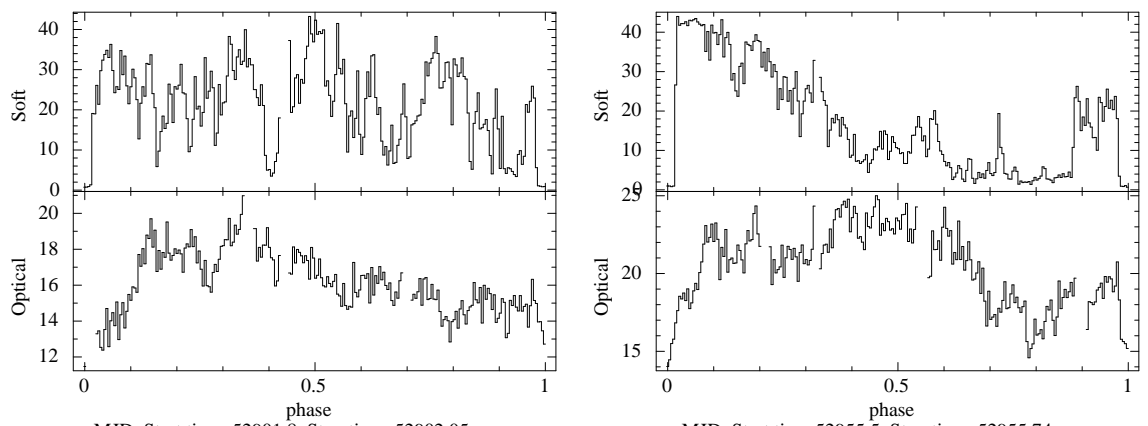

Figure 4: Soft X-ray and corresponding optical variations are classified into five broad classes of orbital profiles. 1) A broad pre-eclipse X-ray absorption between phases 0.6-0.9 (top-left panel). 2) A broad absorption feature around phase 0.2 (top-right panel). 3) A broad absorption feature around phase 0.4 (middle panel). 4 ) Multiple narrow less intense dips at different orbital phases (bottom-left panel). 5) Intense absorption during significant portion of the orbit (bottom-right panel). 
The third is the long term variability at time-scales longer than the above two. To probe this, each orbital segment from the soft and hard X-ray light curve, measured from eclipse to eclipse, was cross-correlated with the simultaneous optical orbital light curve segment with a bin time of $60 \mathrm{~s}$. The soft and hard Xrays were also cross-correlated similarly. Data gaps have been ignored. The thermonuclear X-ray bursts, reprocessed optical bursts and the eclipses were also not included in the cross correlation analysis.

The time delay between the various light curve segments in the three bands were computed using the XRONOS tool crosscor which uses the FFT algorithm. The cross correlation profiles have been classified broadly into five classes solely on the basis of their delays and profile shapes and do not correspond to the variable dipping classification of the soft X-ray light curves, indicated in the previous section. The cross-correlations have been computed for sample light curves, one, from each of the five classes. The CCF profiles (Figure 5, left) are plotted along with the corresponding orbital light curve segment for the hard X-ray, soft X-ray and optical (Figure 5, right). We find that there is little or no correlation between the optical and X-rays at short timescales, and the repeating patterns of the $\mathrm{CCF}$ are independently related to the orbital period (a few 1000s of seconds), being evident from the CCF peaks.

\subsection{Spectral analysis}

We have also carried out a representative study of intensity resolved spectra of the second orbital cycle from the ObsID 0160761301 (Figure 6). We refer to Díaz Trigo et al. (2006) for a more detailed intensity resolved spectral analysis. We extracted the EPIC-PN spectra in the 0.5 - $10 \mathrm{keV}$ energy band from a region of 40 " centered on the source. Segments from the light curve with a count rate less than 20 counts/sec in the $0.5-10 \mathrm{keV}$ band were identified as dipping and the remaining nearly-steady segment was considered as persistent emission. The responses were generated using the SAS task arfgen. Spectral fitting was performed using XSPEC version 12.8.1.

We simultaneously fit the persistent and dip spectra with a powerlaw model along with neutral hydrogen absorber and a partial covering ionized absorber (zxipcf in XSPEC). To avoid the $\mathrm{nH}$ parameter taking unreasonably low values, we fixed it to $0.11 \mathrm{x}$ $10^{22} \mathrm{~cm}^{-2}$, as similarly considered by Díaz Trigo et al. (2006). Strong residuals at $0.569 \mathrm{keV}$ and $0.915 \mathrm{keV}$ were observed, which we then fit using two gaussians. The phabs*zipcf*po + phabs*(gaus+gaus) model in XSPEC gave a reduced $\chi^{2}$ of 1.22 with 319 dof. Table 3 details the best fit parameters for the spectra. We observe an increase in the covering fraction of the ionized absorber and a decrease in the ionization parameter as we move from persistent emission to dipping, as was also reported by Díaz Trigo et al. (2006).

\section{Discussion}

\subsection{Summary of results}

We have studied simultaneous X-ray and optical light curves of EXO 0748-676 from the EPIC-PN detector and the OM from a set of XMM-Newton observations carried out during a hard spectral state of the source. A total of 36 simultaneous and complete, X-ray and optical orbital light curves have been studied. The X-ray and optical dips occur at many different orbital phases with varying depths and widths throughout all the 7 observations. The soft X-ray and the optical emission are seen to vary enormously at sub-orbital time scale and also between successive binary orbital cycles. The non-burst, non-eclipsing $\mathrm{X}$-rays (soft and hard) and optical emission do not show any covariability at the reprocessing time-scales, but show a weak correlation at time-scales of a few 1000s of seconds. EXO 0748676 has been extensively studied in both X-rays and optical in the past. The nature of its X-ray and optical co-variability has been known to change over the years. Our results provide further indications to the evolving nature of the accretion disk in this source.

\subsection{Variability In $X$-rays (soft and hard)}

During the observations reported here, EXO 0748-676 had an average unabsorbed X-ray flux of $2.25 \times 10^{-10} \mathrm{erg} \mathrm{cm}^{-2} \mathrm{~s}^{-1}$ (Boirin et al., 2007), which is nearly $1 \% \mathrm{~L}_{E d d}$. This persistent flux, representative of the mass accretion rate of the neutron star, has been one of the lowest values ever measured for this system before it went into quiescence (Hynes and Jones, 2009). The system was in an X-ray "faint-hard" state throughout all the 7 observations reported here. However, the soft X-ray light curves, excluding the thermonuclear bursts and residual eclipse emission, have a large rms in their variability unlike the hard $\mathrm{X}$-ray light curve variability, which is lower, indicating an unvarying accretion rate and persistent hard X-ray emission from the neutron star.

The soft X-ray light curves, in addition to the eclipses and the bursts, showed intense dipping over a range of orbital phases. The dipping behavior, in it's depth, continuity, orbital phase range, etc. changed considerably from orbit to orbit. A few hard X-ray dips are coincident with strong soft X-ray dips; Also, the absorbing efficiency of the dips is more pronounced at lower energies. The duration of the X-ray dips allow us to roughly estimate the azimuthal angular extent of the absorbing material. As can be seen in Figures 2, 3 and 4, the duration of the dips have a large variation: multiple very narrow dips (as short as $1 \%$ of the orbital period) to one or two wide dips (as long as $\sim 40 \%$ of the orbital period). The soft X-ray dips therefore indicate simultaneous presence of multiple vertical structures with narrow (a few degree) to broad $\left(\sim 150^{\circ}\right)$ azimuthal extent.

EXO 0748-676 has shown a bimodal behavior of transitioning between the hard and soft state in X-rays, while having lowto-moderate accretion rates $\left(0.01-0.5 \mathrm{~L}_{e d d}\right)$. Figure 7 shows the RXTE/ASM light curve indicating the epochs of two XMMNewton observations, one during a bright soft spectral state in 2005 and another during a faint 2003 (this work) hard spectral state. Excluding the thermonuclear bursts, the 2003 observation shows an average of 25 counts/s, while the soft state 2005 observation has a count rate that is about an order of magnitude 

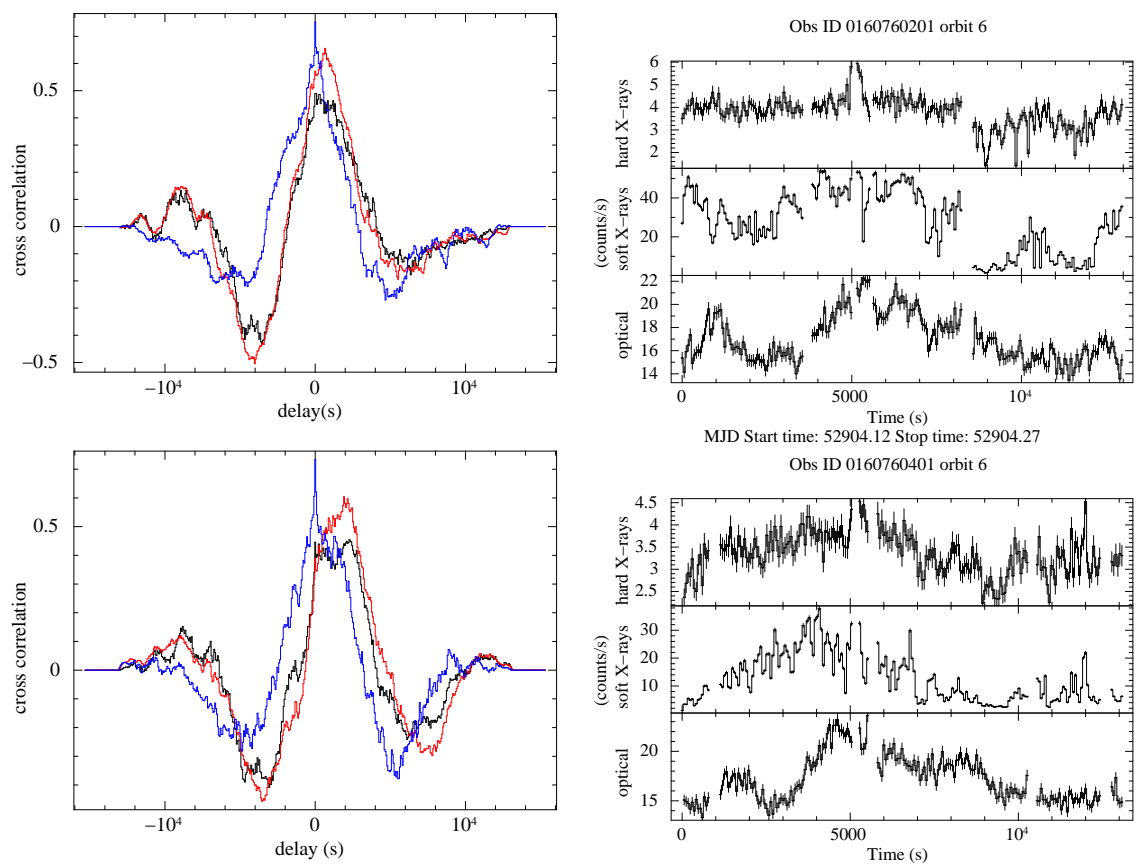

Obs ID 0160760401 orbit 6
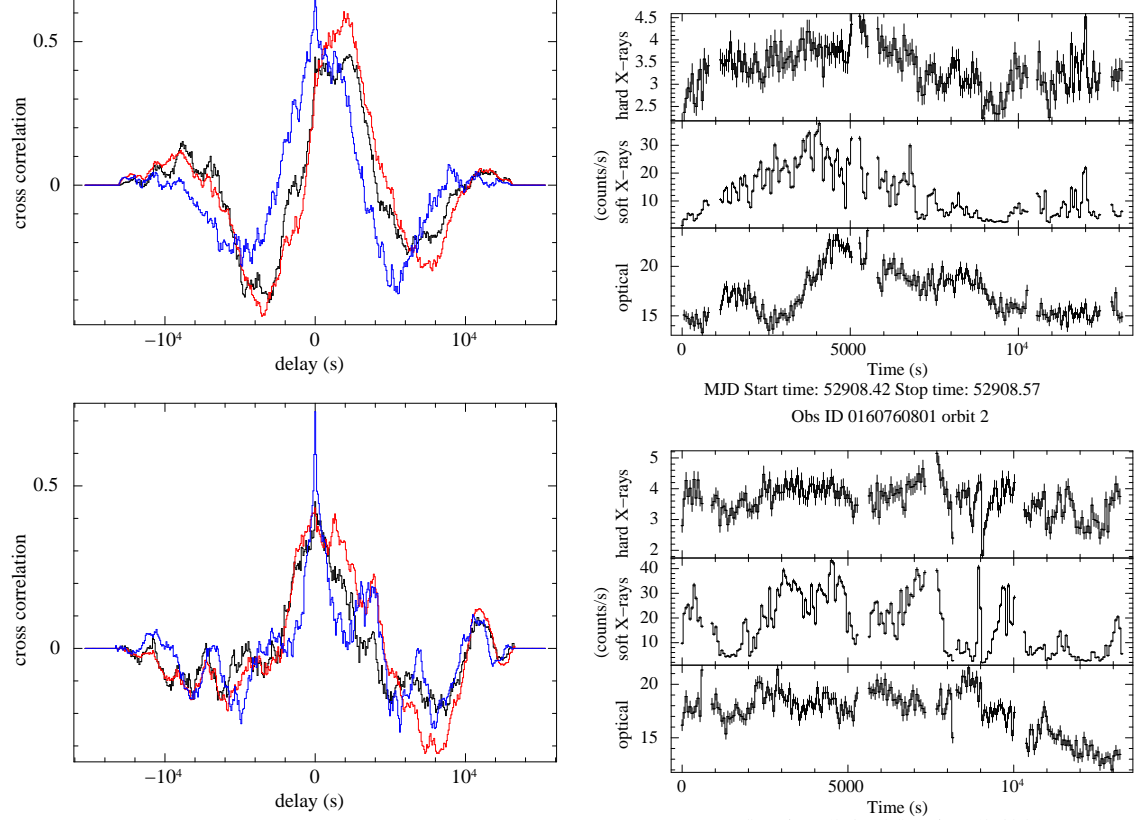
Obs ID 0160760801 orbit 2
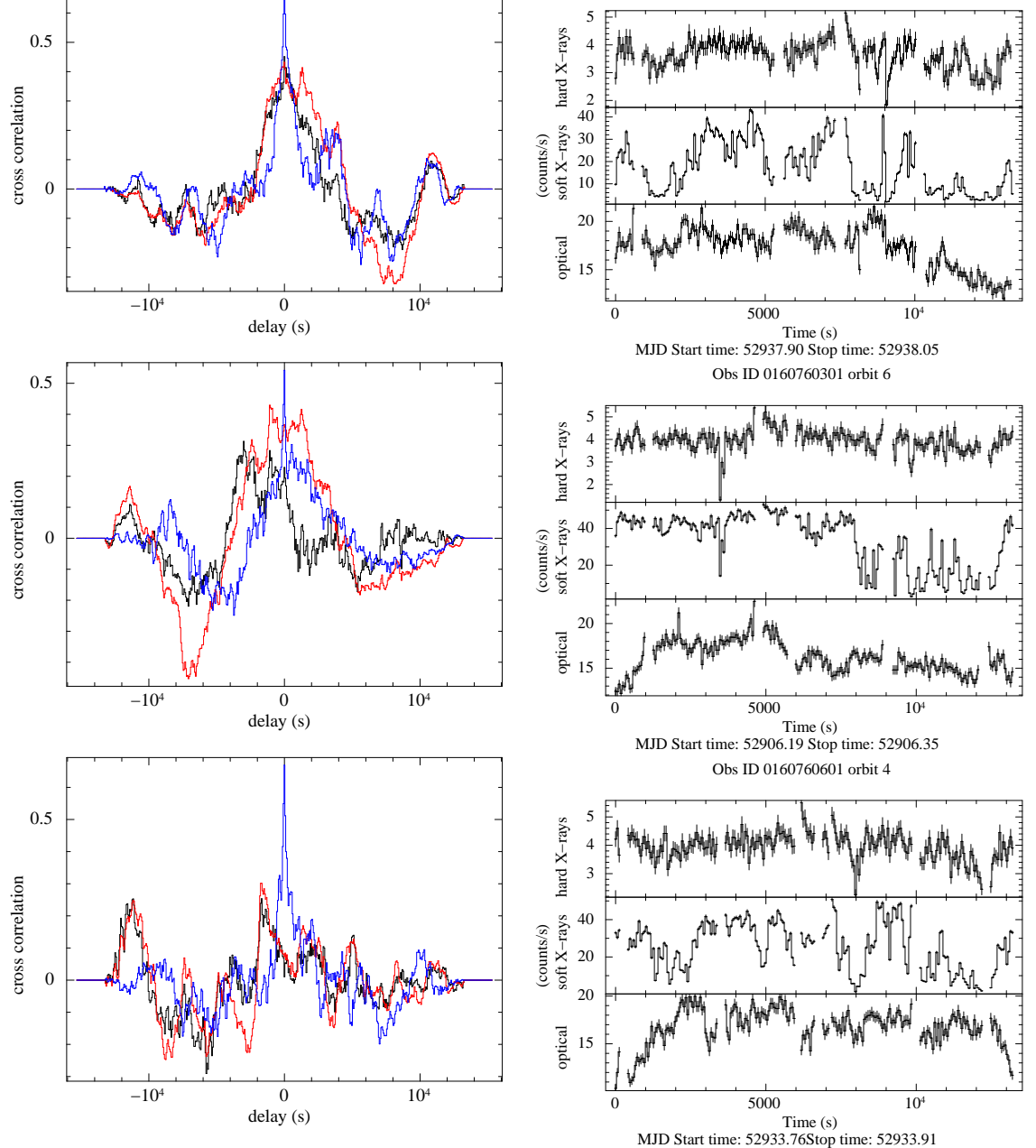

Figure 5: Five different classes of cross correlation analysis patterns between the soft X-ray and optical (red), the hard X-ray and optical (black) and the soft and hard X-ray (blue) have been identified and are shown in the left segment along with the corresponding light curve segments on the right. 


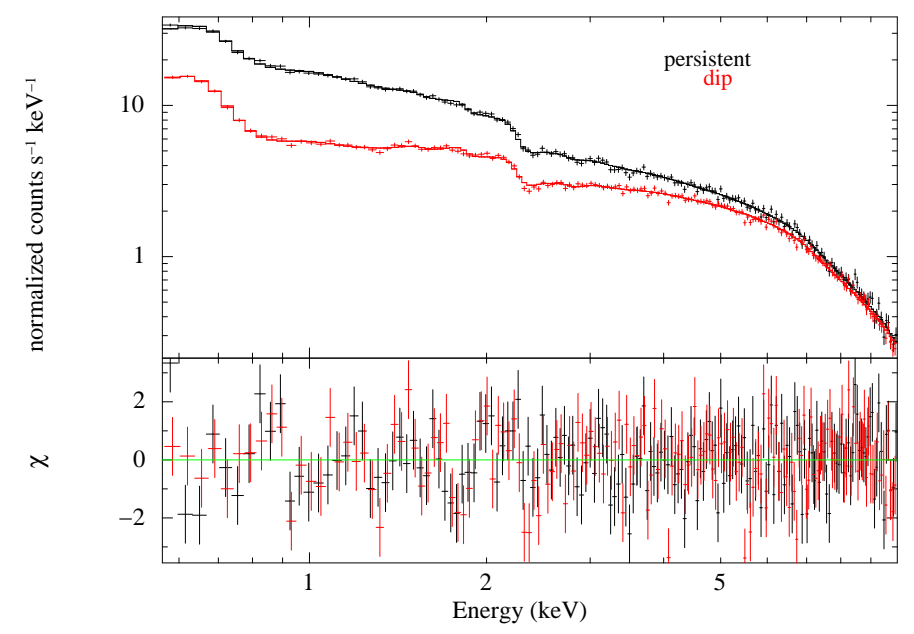

Figure 6: Simultaneous persistent and dipping spectra in the 0.5-10 keV band are shown for the second orbital cycle of Obs-ID 0160761301.

\begin{tabular}{|c|c|c|c|c|}
\hline Parameter & model & Persistent & & Dip \\
\hline $\mathrm{nH}\left(\mathrm{x} 10^{22} \mathrm{~cm}^{-2}\right)$ & phabs & & 0.11 (frozen) & \\
\hline $\mathrm{nH}\left(\mathrm{x} 10^{22} \mathrm{~cm}^{-2}\right)$ & zxipcf & $4.33_{-0.97}^{+0.43}$ & & $4.53_{-0.57}^{+0.45}$ \\
\hline $\log (\xi)$ & zxipcf & $1.39 \pm 0.2$ & & $1.09_{-0.29}^{+0.17}$ \\
\hline cov. f & zxipcf & $0.55_{-0.07}^{+0.03}$ & & $0.78 \pm 0.02$ \\
\hline$\alpha$ & power law & $1.52_{-0.09}^{+0.07}$ & & $1.38 \pm 0.05$ \\
\hline $\mathrm{E}(\mathrm{keV})$ & gaus & & 0.569 (frozen) & \\
\hline $\mathrm{EW}(\mathrm{eV})$ & gaus & $437.2 \pm 0.001$ & & $666.9 \pm 0.0002$ \\
\hline $\mathrm{E}(\mathrm{keV})$ & gaus & & 0.915 (frozen) & \\
\hline EW $(\mathrm{eV})$ & gaus & $70.5 \pm 0.0005$ & & $49.6 \pm 0.0002$ \\
\hline Reduced $\chi^{2}$ & & & 1.22 for 319 d.o.f & \\
\hline
\end{tabular}

higher. Ponti et al. (2014) showed that the dipping phenomenon in the $0.5-5 \mathrm{keV}$ band appears less intense in soft state compared to the hard state. The dips in the soft state are shallow, being almost $75 \%$ of the average count rate. The hard state dips on the other extend much deeper, going down to $~ 33 \%$ of the average count rate (see Figure 8).

Interestingly, light curves from an XMM-Newton observation in 2003, studied earlier by Díaz Trigo et al. (2006) and Boirin et al. (2007), which have also been analyzed in the present paper, show that the dips have widely different characteristics, including some appearing after the eclipse (Obs-IDs 0160760601 and 0160760801, Figures 2, 3 and 4), even when the source is in the same hard spectral state.

The soft X-ray photons, get absorbed by disk structures at different azimuthal angles, due to which they display phasedependent dipping behavior. The structures in the accretion disk that cause the dips can have various origins like irradiation induced vertical structure or warps, stream-impact bulge, ionized absorbers etc., which we describe in detail below.

\subsubsection{Irradiation induced vertical structures}

Irradiation effects are generally invoked in disk accreting
1982) and 4U 1916-05 (Callanan et al., 1995; Homer et al., 2001).

These effects could lead to large scale height and non-axisymmetric vertical structures. These structures can explain the soft Xray modulations seen at different orbital phases (Hakala et al., 2005). Radiation-driven accretion disk warps can lead to instabilities that would produce many of the observed modulations for LMXBs (Pringle, 1996). Warped, precessing accretion disks enable irradiation of outer disk portions in an azimuthally asymmetric way (Clarkson et al., 2003). For Neutron star X-ray binaries, warping effects get prominent after $\sim 3 \times 10^{8}$ $\mathrm{cm}$ and thus affect the outer regions of accretion disks (Pringle, 1996). Although Ogilvie and Dubus (2001) showed that not all LMXBs are likely to experience warping, there is strong observational evidence in our case for some form of disk structures that must be present above the disk plane. Kotze et al. (2009) analyzed the long term RXTE ASM light curve of EXO 0748-676 and carried out a period search using the Lomb Scargle method and reported a super-orbital period corresponding to $180.8 \pm 0.3 \mathrm{~d}$ during the "active" state. They interpret this super-orbital periodicity as a consequence of coupled precessional and warping effects. The current dataset is obtained from a nearly 60 day long observing campaign and hence is not sufficient to look for super-orbital period signatures. Furthermore, Homan et al. (2015) state that origin of the $1 \mathrm{mHz}$ low freLMXB systems (de Jong et al., 1996) like 4U 1822-371 (Mason and (10urow PO in EXO 0748-676 could be geometrical in nature, 


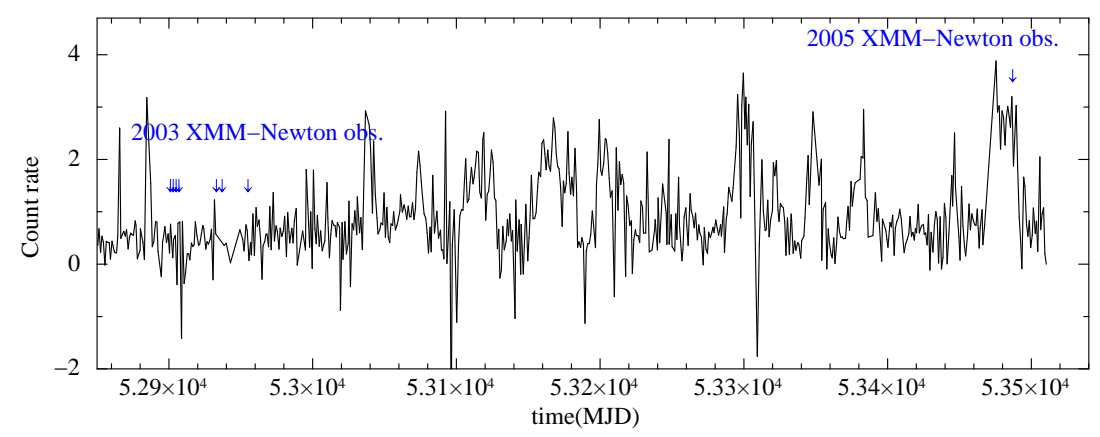

Figure 7: The RXTE-ASM light curve of EXO 0748-676 showing the high-intensity 2005 soft state and low-intensity 2003 (our current dataset) hard state, marked with arrows. These very different X-ray states also show up in the spectral properties of the source.
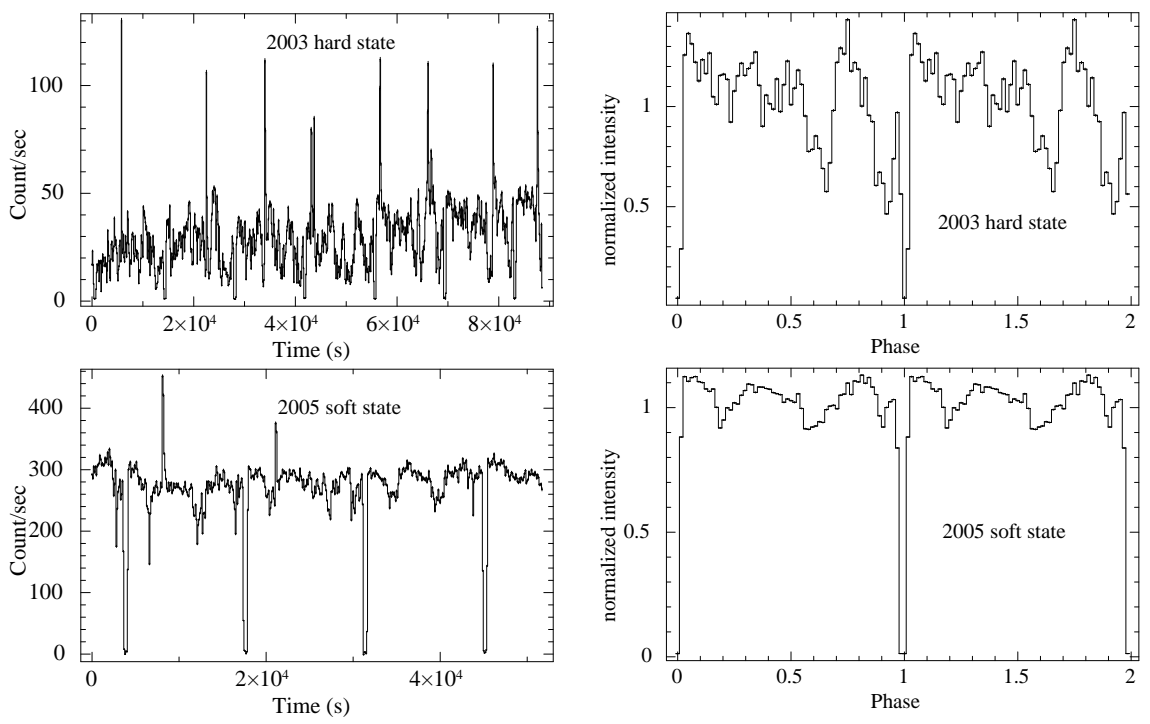

Figure 8: XMM-Newton light curves (2.5 - $10.0 \mathrm{keV}$ ) with a binning of $100 \mathrm{~s}$ and normalized average orbital profiles for the 2003 Obs-ID 0160760101 (top) and the 2005 Obs-ID 0212480501 (bottom) are shown. The 2005 soft state folded profile shows less intense X-ray dips as compared to the more intense dips when the source was in a hard state in 2003.

possibly a misaligned, precessing inner accretion flow.

\subsubsection{Stream-impact bulge at the outer accretion disk}

Changing aspects of the X-ray heated regions of the disk like the stream-impact plasma bulge at the outer disk and/or the ionized plasma above the disk may also contribute to the soft X-ray variability at different orbital phases. They could be responsible for blocking the direct view of the X-ray emitting region, which could cause $\mathrm{X}$-ray dips at different orbital phases.

\subsubsection{Ionized absorber}

Ponti et al. (2014) showed that the presence of a large ionized absorbing component, reduces the opacity, which causes less intense X-ray dips in the soft state compared to the hard state (Figure 8). To explain the nature of the ionized absorber responsible for the dips, two strikingly contrasting models, exist. One involves complex continuum models (Church et al., 1998; Church and Balucinska-Church, 1995) and the other considers simple ionized absorbers (Díaz Trigo et al., 2006). The first approach by Church and Balucinska-Church (1995) assumes the X-ray emission originates from a point-like blackbody together with an extended corona, where the dipping spectra are modeled by partial or progressive covering of the extended corona. However, Díaz Trigo et al. (2006) self-consistently demonstrated that changes in the properties of an ionized absorber provide an alternative and satisfactory explanation for the overall spectral changes during dips for EXO 0748-676 using the current XMM-Newton dataset Obs ID 0160761301, without having to invoke partial or progressive covering models.

A detailed spectral study of this observation, considering all the dips, by Díaz Trigo et al. (2006) revealed that the changes in the spectral continuum from persistent to dipping intervals, reflected in a significant increase in the absorption column density of the ionized absorber upto a factor of 5 and a decrease in the ionization parameter $\log (\xi)$ by $\sim 0.2$. Their study indicates that, in EXO 0748-676, which shows sharp and deep dips, the absorber is significantly less ionized and relatively $\operatorname{cool}(\log (\xi)<2.5)$ unlike other LMXB dipping sources with more ionized absorbers. 


\subsection{Lack of correlated variability between $X$-rays and optical}

\subsubsection{Reprocessing}

Global optical/NIR and X-ray correlation studies conducted in many low magnetic field $\left(\mathrm{B} \leq 10^{11} \mathrm{G}\right.$ ) neutron star LMXBs by Russell et al. (2006) showed that the optical emission can be accounted for by thermal emission due to reprocessing except in cases where the source is in a hard state and its luminosity is very high $\left(>10^{37} \mathrm{ergs} / \mathrm{s}\right)$. In such a scenario, synchrotron emission from jets contributes heavily to the NIR and optical emission. However, since X-ray reprocessing usually dominates the optical emission for lower luminosities, which is the current case, the X-ray spectral state changes should reflect in the optical emission for this binary.

\subsubsection{Burst reprocessing}

Simultaneous X-ray and optical observations of thermonuclear X-ray bursts in LMXBs are very useful in understanding the X-ray reprocessing phenomena and also to investigate the relative importance of the two processes of optical emission: X-ray heating and viscous dissipation. Burst reprocessing into optical emission has been observed in many LMXB sources and is an expected consequence of interception of a fraction of the primary burst X-ray photons by the disk and/or companion surface. Dubus et al. (1999) showed that a point source cannot irradiate a planar (non-warped) stationary disk because self-screening would prevent the outer parts from getting affected by irradiation. A non-irradiated disk model is incapable of explaining the optical emission from a large fraction of persistent LMXBs. A concave disk (a consequence of irradiation), extending to larger scale heights as we move outwards (de Jong et al., 1996) would ensure the outer disk intercepts some part of the X-rays. In the case of EXO 0748-676, the delays between the X-ray and optical bursts are consistent with the binary separation (Hynes et al., 2006; Paul et al., 2012). Smearing in the delays, also indicates a finite extent of this reprocessing region which is most likely present at the outer accretion disk. Reprocessing of the thermonuclear X-ray bursts therefore show that since a significant part of the burst X-ray emission is reprocessed in the outer accretion disk, same may hold true for the non-burst emission.

\subsubsection{Non-burst reprocessing fraction}

Considering a conversion factor for the non-burst reprocessing phenomenon, similar to the burst emission, roughly $24 \%$ of the total optical emission can be assumed to be due to the reprocessing of X-rays. However, this is a lower limit, and a larger fraction of the optical emission is probably due to X-ray reprocessing. The X-ray emission from LMXBs is anisotropic and is enhanced in the direction perpendicular to the disk plane (Fujimoto, 1988; He and Keek, 2016). The persistent X-ray emission tends to have a higher degree of anisotropy compared to the burst emission. Thus, for high inclination systems like EXO 0748-676, the observable X-ray to optical conversion ratio of the non-burst emission would be higher than the burst emission. The residual optical emission during the X-ray eclipses is partly from the companion star and partly from the un-eclipsed disk. Considering an average source magnitude of $\sim 17$, the optical flux in the V-band is $\sim 4.1 \times 10^{-13} \mathrm{erg} \mathrm{cm}^{-2} \mathrm{~s}^{-1}$ and the 1 $10 \mathrm{keV} \mathrm{X}$-ray flux is $2.25 \times 10^{-10} \mathrm{erg} \mathrm{cm}^{-2} \mathrm{~s}^{-1}$. This gives an $\mathrm{X}-$ ray to $\mathrm{V}$-band optical flux ratio of $\sim 550$ and a reprocessing efficiency from X-ray flux to V-band optical flux of $\sim 0.044 \%$. Assuming the companion in EXO 0748-676 to be a typical M2V type star with radius of $0.44 \mathrm{R}_{\odot}$, a surface temperature of 6000 $\mathrm{K}$ (Hynes and Jones, 2009) and a source distance of $5 \mathrm{kpc}$, the total optical flux is $\sim 2.7 \times 10^{-13} \mathrm{erg} \mathrm{cm}^{-2} \mathrm{~s}^{-1}$. The V-band optical flux of the companion star is thus a small fraction of the total source emission in the V-band mentioned above.

\subsubsection{Optical eclipses/dips correlations with X-rays}

The optical eclipses of EXO 0748-676 are not total, and are broader compared to the X-ray eclipses. This indicates that a part of the optical emission is from a region that is centered around the X-ray source, but larger, perhaps with a size comparable to the size of the companion star. A non-zero optical intensity during the eclipse indicates a considerable emission from a broader region, perhaps the outer disk and a small contribution from the companion. Although the broad optical eclipses do coincide with the X-ray eclipses, a number of other variabilities (like narrow or wide dips) are not particularly coincident with the soft X-ray dips. The CCFs display variable positive soft X-ray to optical as well as hard X-ray to optical delays at time scales of a few $1000 \mathrm{~s}$ for almost all the orbital profiles. The cross-correlation coefficients are small in all the 7 observations. Also, the CCFs are variable from orbit-to-orbit in delays and strength. The lack of correlation at reprocessing time-scales is expected and is consistent with the correlation analysis results. However, the observed weak correlation, seen in the repeating patterns of the $\mathrm{CCF}$, that exists at sub-orbital time-scales of a few 1000s of seconds, is probably related to orbital period. This time scale is different than the dynamic or viscous time-scales in the binary and can possibly arise due to variable outer accretion disk structures, discussed in section 4.1.

We also note that we were unable to compare the X-ray optical co-variability of the source during the hard spectral state (2003) with the soft spectral state (2005), since the OM data from the XMM-Newton observation in 2005 (Obs-ID 0212480501) was of a poor statistical quality.

\subsubsection{Large optical variability}

The input for the reprocessing phenomena, the X-rays from the compact star is likely to be stable since the mass accretion rate and luminosity of the source is fairly constant during the entire duration of all the 7 observations (Boirin et al., 2007). The optical emission from a stable accretion disk with fairly constant mass accretion rate must have a smooth orbital modulation. It is unexpected to show changes in shape as shown in Figure 3. The soft X-ray variability is due to absorption by structures in the accretion disk. However, emission from the outer disk will have variability that is different from the X-rays. Vertical accretion disk structures like the ones addressed in section 4.1 can produce orbital optical intensity variation both due 
to changing visibility of the structures themselves or the visibility of their X-ray reprocessing faces.

\subsection{Mean optical intensity and optical states}

The optical counterpart of EXO 0748-676 is known to exhibit high and low intensity states similar to those defined for the X-ray (Motch et al., 1989b). The high state corresponds to a high mean optical intensity which also exhibits a broad minima at the phases $0.6-1.2$. The low state, on the other hand exhibits a lower mean optical intensity with the broad optical dip disappearing and the sinusoidal shape flattening out (Thomas et al., 1993). In the current dataset, the optical light curves of Obs-IDs 0160760101, 0160760201, 0160760301 and 0160760401 show a broad minima, with some orbits showing peaks, and the shape seen in Obs-IDs 0160760601,0160760801 and 0160761301 is different: flattened sinusoids without any peaks. Within the current set of 7 observations having similar orbital averaged optical intensity, the shape of the optical modulation varied between what was earlier known to be associated with the optical bright and low intensity states, indicating that the optical orbital intensity profiles are not intrinsically related to the mean optical intensity.

\section{Conclusions}

From simultaneous X-ray and optical observations of EXO 0748-676 in the hard spectral state, we conclude that there is no co-variability between the X-rays and optical light seen in most of the orbital light curves at reprocessing or orbital timescales, but a weak correlation exists at a few 1000s time-scales. There exist vertical structures in the outer accretion disk that evolve at binary orbital time scales of a few hours. Large optical variability between successive orbits could be the result of reprocessing occurring at these evolving disk structures. These structures could be the result of irradiation effects and/or warping. Modeling these effects would require more sophisticated understanding of an irradiated accretion disk and warps, both of which are still at a very nascent stage. More simultaneous $\mathrm{X}$-ray and optical data would improve statistics and help analyze these scenarios better.

Acknowledgements : This work has made use of archival data obtained from the High Energy Astrophysics Science Archive Research Center (HEASARC), provided by the NASA Goddard Space Flight Center.

\section{References}

Boirin, L., Keek, L., Méndez, M., Cumming, A., in’t Zand, J. J. M., Cottam, J., Paerels, F., Lewin, W. H. G., Apr. 2007. Discovery of X-ray burst triplets in EXO 0748-676. A\&A465, 559-573.

Cackett, E. M., Brown, E. F., Degenaar, N., Miller, J. M., Reynolds, M., Wijnands, R., Aug. 2013. An X-ray-UV correlation in Cen X-4 during quiescence. MNRAS433, 1362-1368.

Callanan, P. J., Grindlay, J. E., Cool, A. M., Apr. 1995. Optical Observations of 4 U 1915-05, and the Stability of the Long Term Ephemeris. PASJ47, 153162.
Church, M. J., Balucinska-Church, M., Aug. 1995. A complex continuum model for the low-mass X-ray binary dipping sources: application to $\mathrm{X}$ 1624-49. A\&A300, 441.

Church, M. J., Bałucińska-Church, M., Dotani, T., Asai, K., Sep. 1998. Progressive Covering of the Accretion Disk Corona during Dipping in the LowMass X-Ray Binary XBT 0748-676. ApJ504, 516-521.

Clarkson, W. I., Charles, P. A., Coe, M. J., Laycock, S., Aug. 2003. Long-term properties of accretion discs in X-ray binaries - II. Stability of radiationdriven warping. MNRAS343, 1213-1223.

Corbet, R. H. D., Smale, A. P., Charles, P. A., Lewin, W. H. G., Menzies, J. W., Naylor, T., Penninx, W., Sztajno, M., Thorstensen, J. R., Trumper, J., van Paradijs, J., Aug. 1989. Connections between X-ray and optical variability in the low mass X-ray binary 1735-444. MNRAS239, 533-547.

Cottam, J., Paerels, F., Méndez, M., Boirin, L., Lewin, W. H. G., Kuulkers, E., Miller, J. M., Jan. 2008. The Burst Spectra of EXO 0748-676 during a Long 2003 XMM-Newton Observation. ApJ672, 504-509.

Crampton, D., Stauffer, J., Hutchings, J. B., Cowley, A. P., Ianna, P., Jul. 1986. Optical observations of the X-ray nova EXO 0748-676 near maximum light. ApJ306, 599-604.

de Jong, J. A., van Paradijs, J., Augusteijn, T., Oct. 1996. Reprocessing of X rays in low-mass X-ray binaries. A\&A314, 484-490.

Degenaar, N., Wolff, M. T., Ray, P. S., Wood, K. S., Homan, J., Lewin, W. H. G., Jonker, P. G., Cackett, E. M., Miller, J. M., Brown, E. F., Wijnands, R., Apr. 2011. Further X-ray observations of EXO 0748-676 in quiescence: evidence for a cooling neutron star crust. MNRAS412, 1409-1418.

Díaz Trigo, M., Parmar, A. N., Boirin, L., Méndez, M., Kaastra, J. S., Jan. 2006. Spectral changes during dipping in low-mass X-ray binaries due to highly-ionized absorbers. A\&A445, 179-195.

Dubus, G., Lasota, J.-P., Hameury, J.-M., Charles, P., Feb. 1999. X-ray irradiation in low-mass binary systems. MNRAS303, 139-147.

Fujimoto, M. Y., Jan. 1988. Angular distribution of radiation from low-mass X-ray binaries. ApJ324, 995-1000.

Galloway, D. K., Lin, J., Chakrabarty, D., Hartman, J. M., Mar. 2010. Discovery of a $552 \mathrm{~Hz}$ Burst Oscillation in the Low-Mass X-Ray Binary EXO 0748-676. ApJ711, L148-L151.

Gottwald, M., Haberl, F., Parmar, A. N., White, N. E., Sep. 1986. The bursting behavior of the transient X-ray burst source EXO 0748-676 - A dependence between the X-ray burst properties and the strength of the persistent emission. ApJ308, 213-224.

Hakala, P., Ramsay, G., Muhli, P., Charles, P., Hannikainen, D., Mukai, K., Vilhu, O., Jan. 2005. XMM-Newton observations of UW CrB: detection of $\mathrm{X}$-ray bursts and evidence for accretion disc evolution. MNRAS356, 11331138 .

He, C.-C., Keek, L., Mar. 2016. Anisotropy of X-Ray Bursts from Neutron Stars with Concave Accretion Disks. ApJ819, 47.

Homan, J., Fridriksson, J. K., Remillard, R. A., Oct. 2015. On the Geometric Nature of Low-frequency Quasi-periodic Oscillations in Neutron-star Lowmass X-Ray Binaries. ApJ812, 80.

Homan, J., Neilsen, J., Allen, J. L., Chakrabarty, D., Fender, R., Fridriksson, J. K., Remillard, R. A., Schulz, N., Jun. 2016. Evidence for simultaneous jets and disk winds in luminous low-mass X-ray binaries. ArXiv e-prints.

Homan, J., van der Klis, M., Aug. 2000. A 695 HZ Quasi-periodic Oscillation in the Low-Mass X-Ray Binary EXO 0748-676. ApJ539, 847-850.

Homer, L., Charles, P. A., Hakala, P., Muhli, P., Shih, I.-C., Smale, A. P., Ramsay, G., Apr. 2001. On the multi-periodicities in the X-ray dipper XB1916053. MNRAS322, 827-842.

Hynes, R. I., Charles, P. A., Garcia, M. R., Robinson, E. L., Casares, J., Haswell, C. A., Kong, A. K. H., Rupen, M., Fender, R. P., Wagner, R. M., Gallo, E., Eves, B. A. C., Shahbaz, T., Zurita, C., Aug. 2004. Correlated XRay and Optical Variability in V404 Cygni in Quiescence. ApJ611, L125L128.

Hynes, R. I., Horne, K., O’Brien, K., Haswell, C. A., Robinson, E. L., King, A. R., Charles, P. A., Pearson, K. J., Sep. 2006. Multiwavelength Observations of EXO 0748-676. I. Reprocessing of X-Ray Bursts. ApJ648, 11561168.

Hynes, R. I., Jones, E. D., May 2009. The Quiescent Optical and Infrared Counterpart to EXO 0748-676 = UY Vol. ApJ697, L14-L17.

Ilovaisky, S. A., Chevalier, C., White, N. E., Mason, K. O., Sanford, P. W., Delvaille, J. P., Schnopper, H. W., Apr. 1980. Simultaneous X-ray and optical observations of rapid variability in Scorpius X-1. MNRAS191, 81-93.

Kotze, M. M., Charles, P. A., Crause, L. A., May 2009. Discovery of long-term 
superorbital periodicities in the pseudo-transient LMXBs: IGRJ17098-3628 and EXO0748-676. MNRAS395, 1579-1584.

Machin, G., Callanan, P. J., Charles, P. A., Thorstensen, J., Brownsberger, K., Corbet, R. H. D., Hamwey, R., Harlaftis, E. T., Mason, K. O., Mukai, K., Nov. 1990. Optical and X-Ray Observations of 4U:0614+09. MNRAS247, 205

Mason, K. O., Breeveld, A., Much, R., Carter, M., Cordova, F. A., Cropper, M. S., Fordham, J., Huckle, H., Ho, C., Kawakami, H., Kennea, J., Kennedy, T., Mittaz, J., Pandel, D., Priedhorsky, W. C., Sasseen, T., Shirey, R., Smith, P., Vreux, J.-M., Jan. 2001. The XMM-Newton optical/UV monitor telescope. A\&A365, L36-L44.

Mason, K. O., Cordova, F. A., Apr. 1982. Ultraviolet spectrophotometry of 2A 1822-371 - A bulge on the accretion disk. ApJ255, 603-609.

Motch, C., Pakull, M. W., Mouchet, M., Beuermann, K., Jul. 1989a. An Xray and optical study of the low-mass X-ray source 4 U 1556-605. A\&A219, $158-166$.

Motch, C., Pedersen, H., Ilovaisky, S. A., Chevalier, C., Mouchet, M., Nov. 1989b. Optical emission from the high inclination $\mathrm{X}$ ray transient EXO 0748-676. In: Hunt, J., Battrick, B. (Eds.), Two Topics in X-Ray Astronomy, Volume 1: X Ray Binaries. Volume 2: AGN and the X Ray Background. Vol. 296 of ESA Special Publication.

Ogilvie, G. I., Dubus, G., Feb. 2001. Precessing warped accretion discs in X-ray binaries. MNRAS320, 485-503.

Parmar, A. N., White, N. E., Giommi, P., Gottwald, M., Sep. 1986. The discovery of 3.8 hour periodic intensity dips and eclipses from the transient low-mass X-ray binary EXO 0748-676. ApJ308, 199-212.

Parmar, A. N., White, N. E., Giommi, P., Haberl, F., Pedersen, H., Mayor, M., Feb. 1985. EXO 0748-676. IAU Circ.4039.

Paul, B., Archana, M., Saripalli, L., Jun. 2012. Simultaneous X-ray and optical observations of thermonuclear bursts in the LMXB EXO 0748-676. Bulletin of the Astronomical Society of India 40, 93-104.

Pedersen, H., Cristiani, S., D’Odorico, S., Thomsen, B., Angebault, P., Mar. 1985. EXO 0748-676. IAU Circ.4047.

Ponti, G., Bianchi, S., Muñoz-Darias, T., De Marco, B., Dwelly, T., Fender, R. P., Nandra, K., Rea, N., Mori, K., Haggard, D., Heinke, C. O., Degenaar, N., Aramaki, T., Clavel, M., Goldwurm, A., Hailey, C. J., Israel, G. L., Morris, M. R., Rushton, A., Terrier, R., Jan. 2015. On the Fe K absorption - accretion state connection in the Galactic Centre neutron star X-ray binary AX J1745.6-2901. MNRAS446, 1536-1550.

Ponti, G., Fender, R. P., Begelman, M. C., Dunn, R. J. H., Neilsen, J., Coriat, M., May 2012. Ubiquitous equatorial accretion disc winds in black hole soft states. MNRAS422, 11-15.

Ponti, G., Muñoz-Darias, T., Fender, R. P., Oct. 2014. A connection between accretion state and Fe $\mathrm{K}$ absorption in an accreting neutron star: black holelike soft-state winds? MNRAS444, 1829-1834.

Pringle, J. E., Jul. 1996. Self-induced warping of accretion discs. MNRAS281, 357-361.

Russell, D. M., Fender, R. P., Hynes, R. I., Brocksopp, C., Homan, J., Jonker, P. G., Buxton, M. M., Sep. 2006. Global optical/infrared-X-ray correlations in X-ray binaries: quantifying disc and jet contributions. MNRAS371, 1334-1350.

Southwell, K. A., O’Donoghue, D., Charles, P. A., Feb. 1996. The optical light curve of the eclipsing LMXB EXO 0748-676. In: Zimmermann, H. U., Trümper, J., Yorke, H. (Eds.), Roentgenstrahlung from the Universe. pp. 191-192.

Thomas, B., Corbet, R., Augusteijn, T., Callanan, P., Smale, A. P., May 1993. Optical and X-ray observations of the low-mass X-ray binary EXO 0748676. ApJ408, 651-655.

van Paradijs, J., van der Klis, M., Pedersen, H., Dec. 1988. Optical photometry of the X-ray source EXO 0748-676/UY Volantis. A\&AS76, 185-188.

Wade, R. A., Quintana, H., Horne, K., Marsh, T. R., Nov. 1985. Optical studies of the X-ray transient EXO 0748-676. PASP97, 1092-1095.

Wolff, M. T., Ray, P. S., Wood, K. S., Hertz, P. L., Jul. 2009. Eclipse Timings of the Transient Low-Mass X-ray Binary EXO 0748-676. IV. The Rossi X-ray Timing Explorer Eclipses. ApJS183, 156-170.

\section{Appendix A. 36 complete simultaneous hard X-ray, soft X- ray and optical orbital light curve profiles.}

EXO 0748-676 was observed during September-November, 2003 with XMM-Newton. A total of 7 observations were carried out covering 36 complete orbital light curve segments, simultaneously in the X-ray and the optical bands. The simultaneous hard X-ray (5 - $10.0 \mathrm{keV})$, soft X-ray (0.3 - 5.0 keV) and optical (white band) light curves are shown for all the 7 Obs-IDs. The thermonuclear bursts have been excluded in order to highlight the non-burst intensity modulation between successive orbital light curve segments. Incomplete orbital segments at the start and end of each Obs-ID are not shown here. 

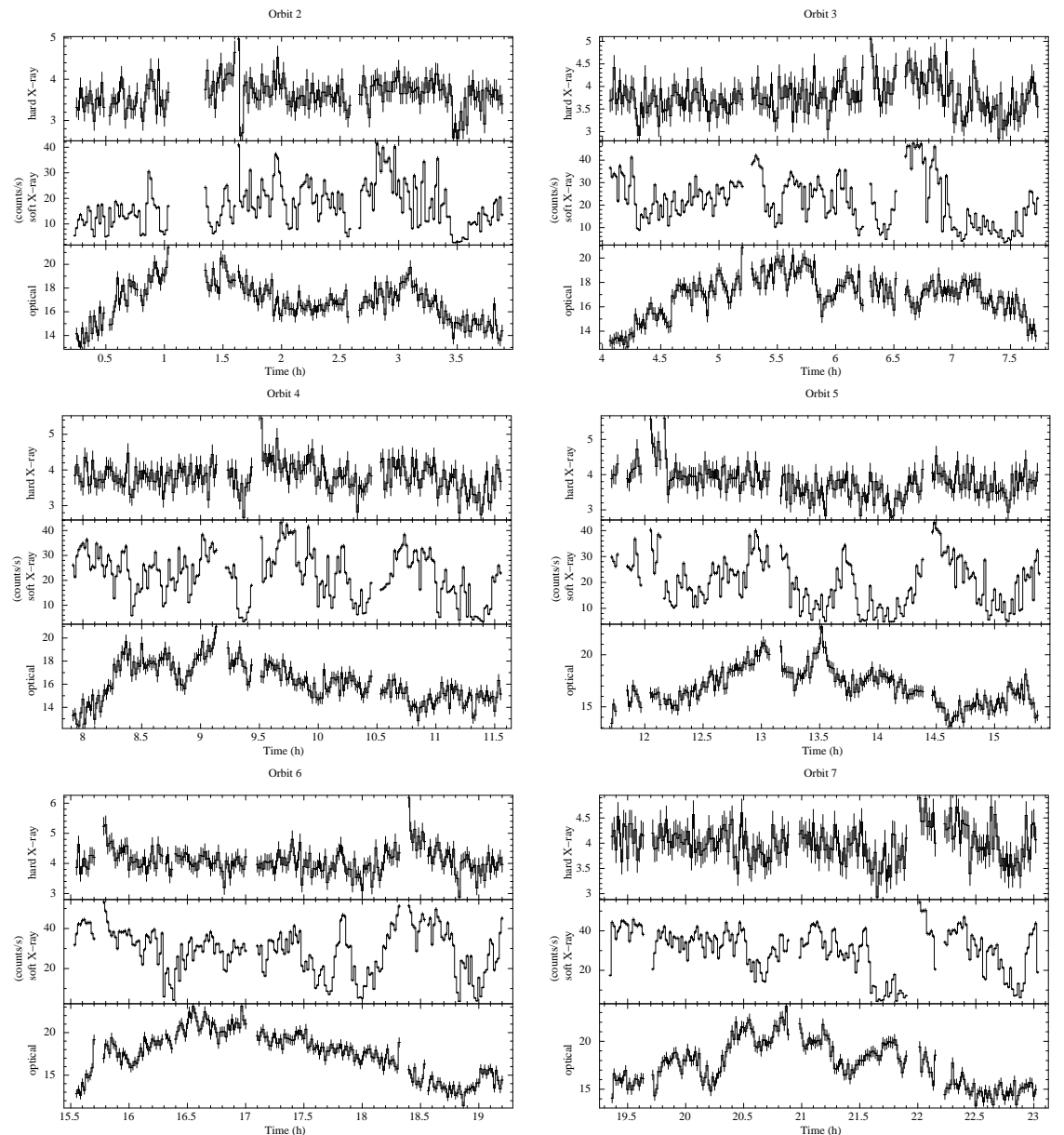

Figure Appendix A.1: Obs-ID 0160760101 

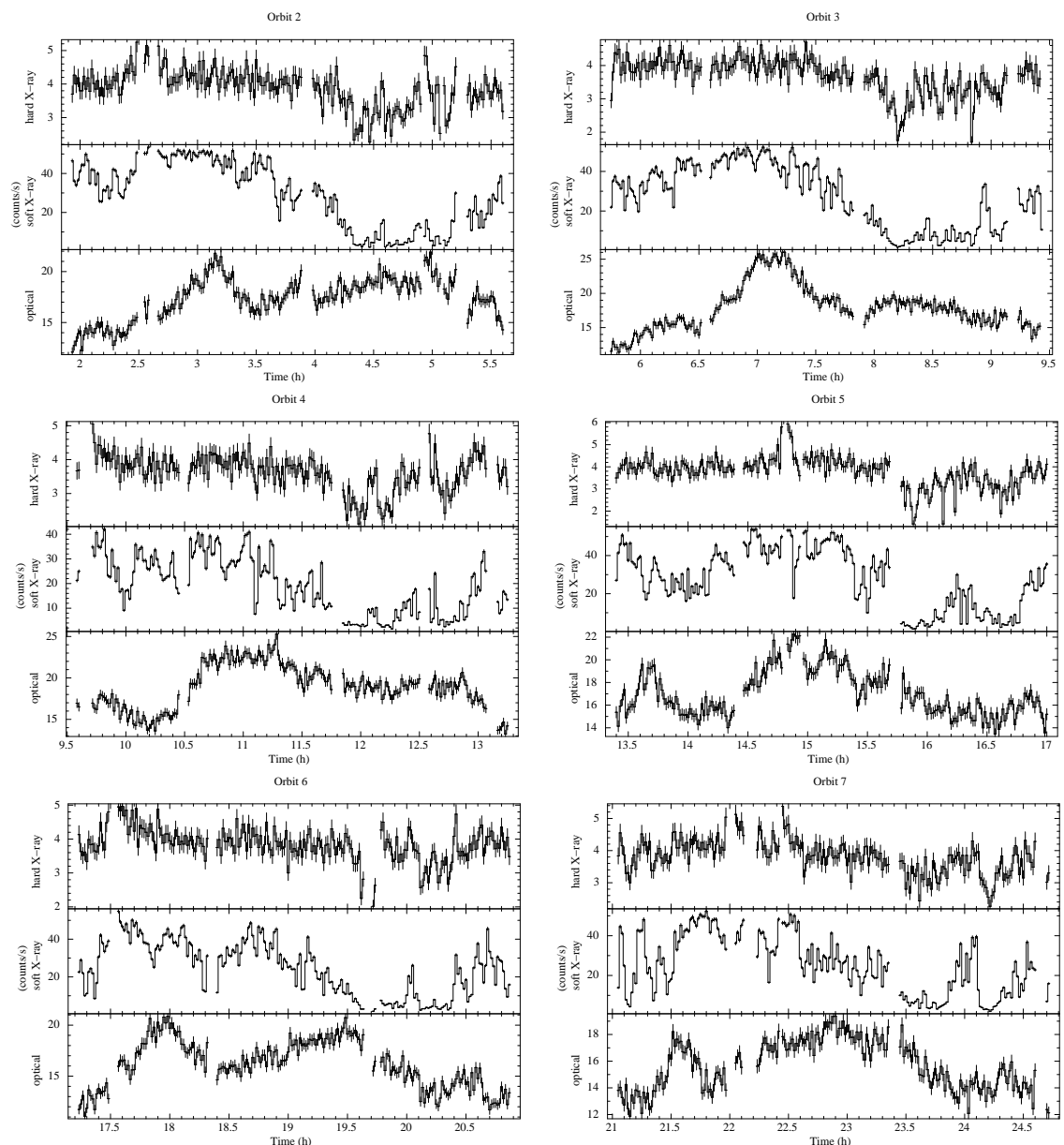

Figure Appendix A.2: Obs-ID 0160760201 

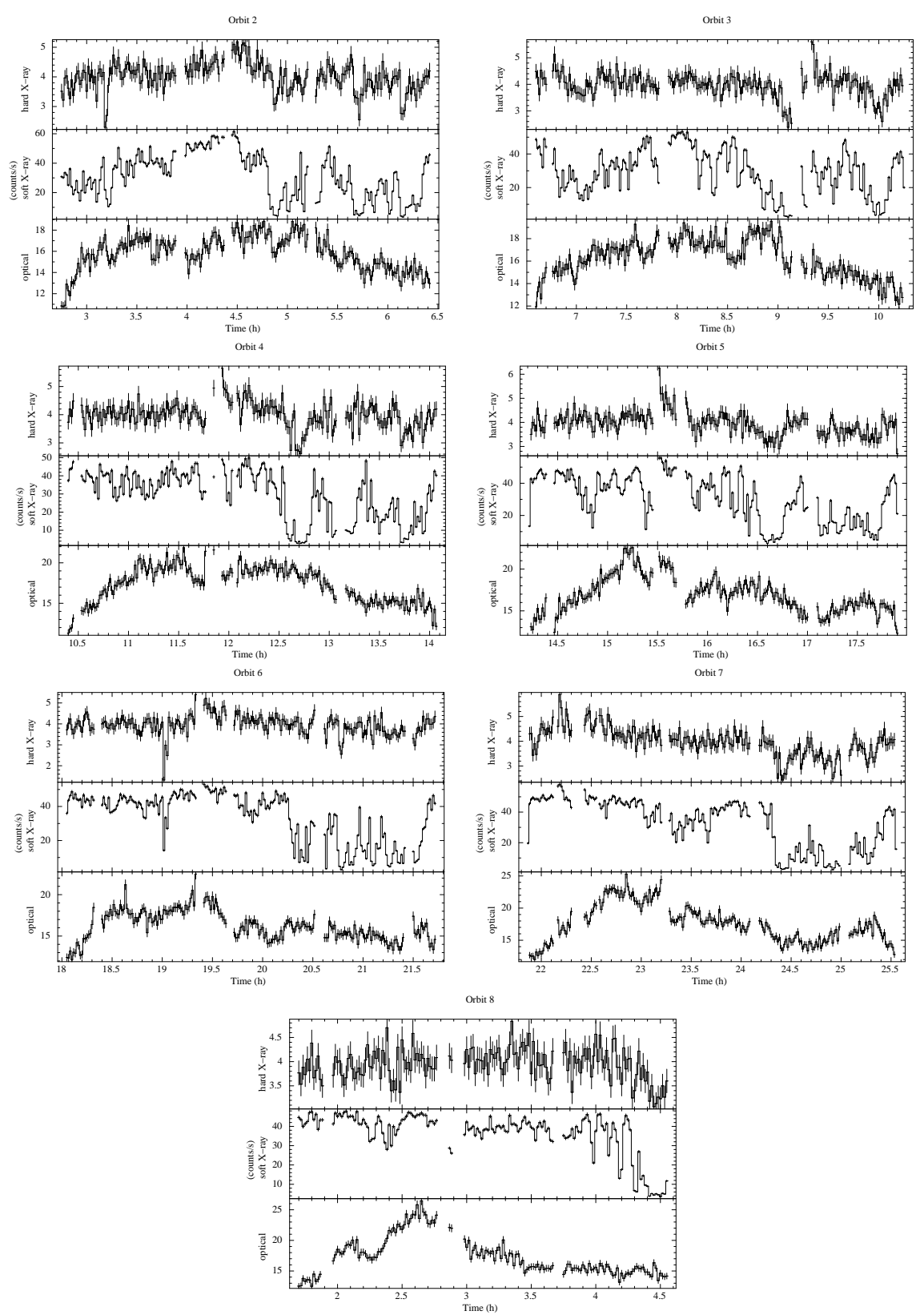

Figure Appendix A.3: Obs-ID 0160760301 

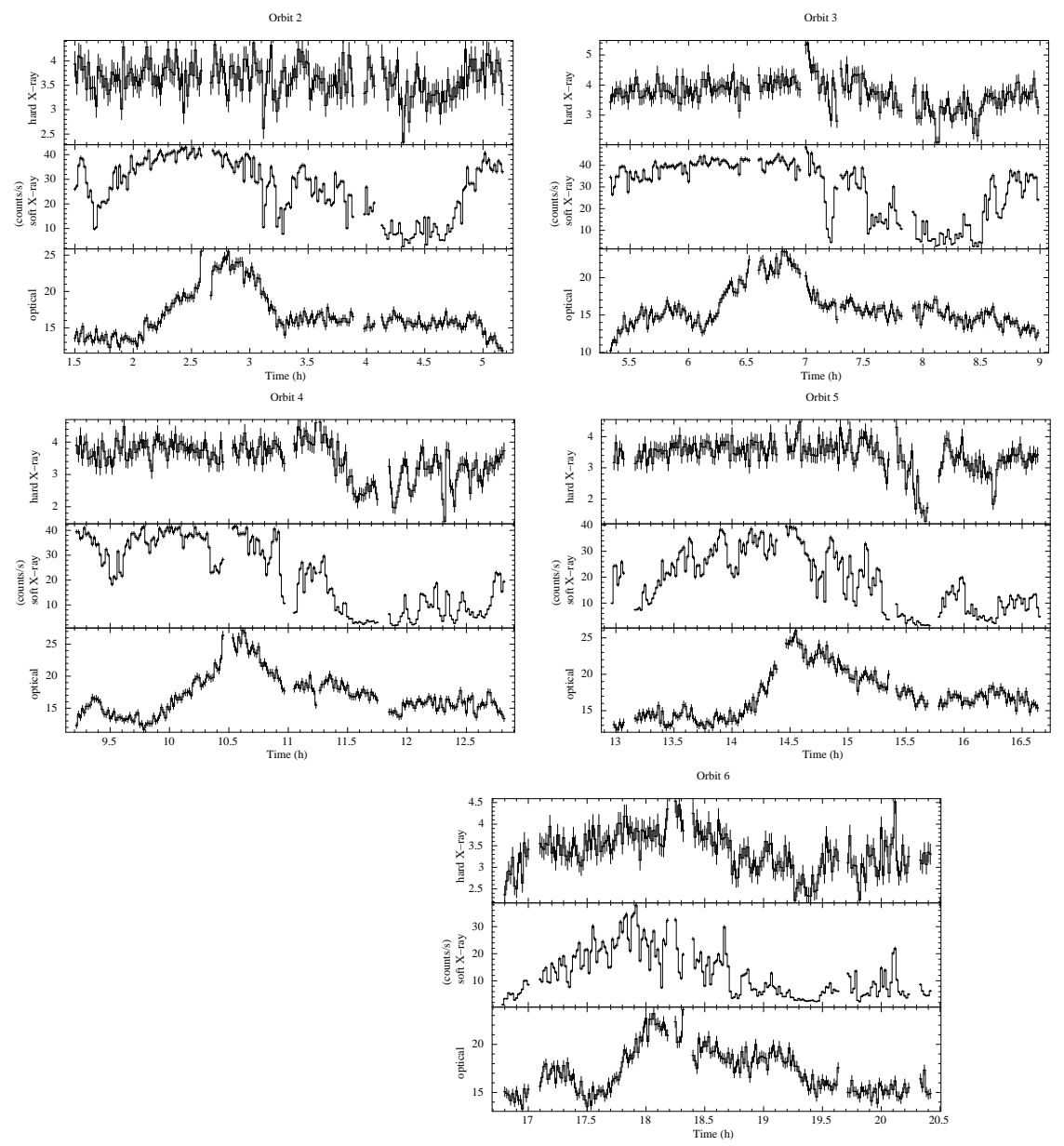

Figure Appendix A.4: Obs-ID 0160760401
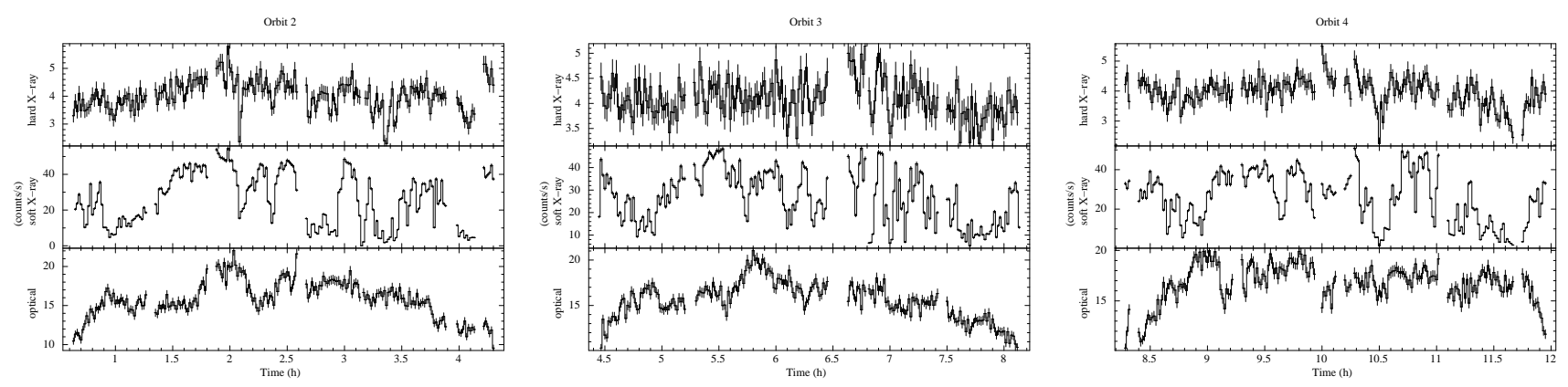

Figure Appendix A.5: Obs-ID 0160760601
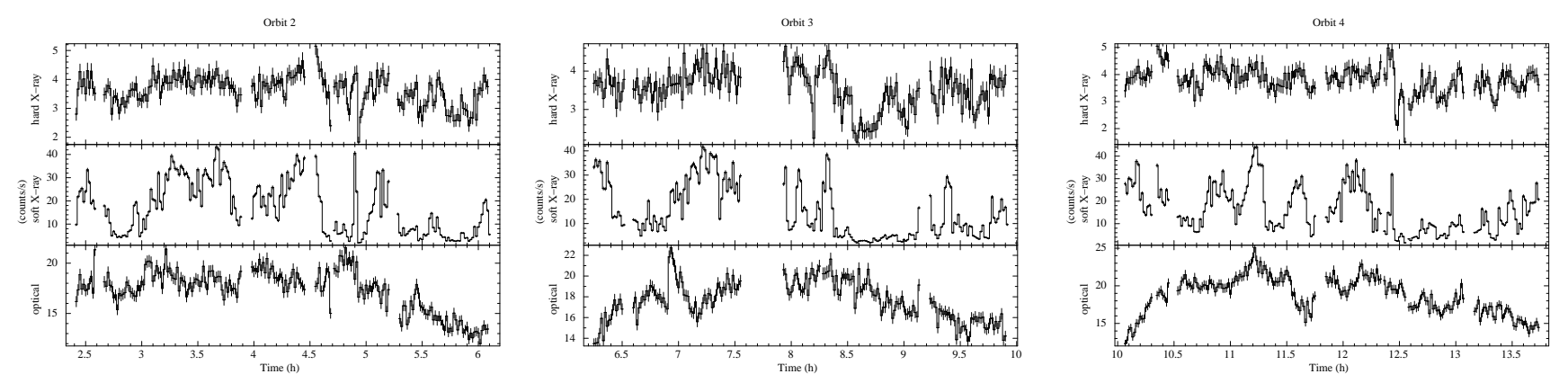

Figure Appendix A.6: Obs Id 0160760801 

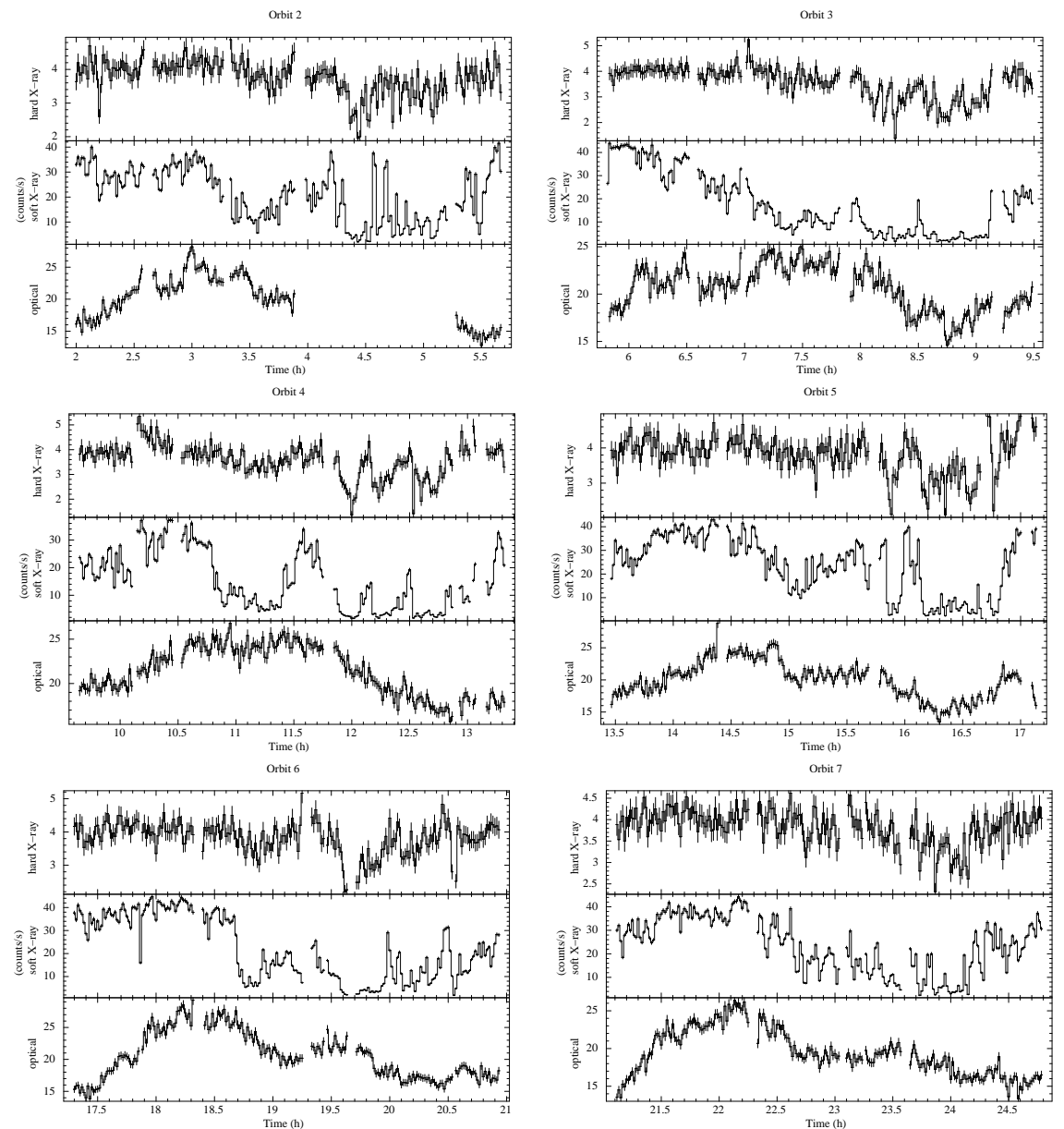

Figure Appendix A.7: Obs-ID 0160761301 